\title{
Primeros datos sobre Myotis alcathoe von Helversen \& Heller, 2001 en Castilla y León y primer refugio de cría localizado en el noroeste de la península Ibérica
}

\author{
Roberto J. Hermida*, Manuel Arzúa, Ledicia Santos y Francisco J. Lamas
}

Morcegos de Galicia - Drosera.

Pdo. Magdalena, G-2, $2^{\circ}$ izquierda, 15320 As Pontes, A Coruña (Spain)

* Correo electrónico del autor: info@morcegosdegalicia.org

DOI: http://dx.doi.org/10.14709/BarbJ.6.1.2013.04

English title: First report of Myotis alcathoe von Helversen \& Heller, 2001 in Castilla y León and first breeding roost in the northeast Iberian Peninsula

\begin{abstract}
First record of Myotis alcathoe is reported for the Autonomous Community of Castilla y Leon. Five individuals were caught in the Ancares mountains and data on roosting behavior is documented by radiotracking them. Two roosts were used out of the breeding season in trees with crevices generated by longitudinal scared process along the trunk. A maternity roost was also found during the study and it represents the first record in the northwestern area of the Iberian Peninsula. The breeding colony was found in a big and almost death Quercus robur.
\end{abstract}

Keywords: Myotis alcathoe, Castilla y Leon, roost, Ancares, Galicia

Resumen: En la presente nota se cita por primera vez la presencia de Myotis alcathoe en la Comunidad Autónoma de Castilla y León. Hemos capturado cinco ejemplares en la sierra de Ancares y hemos obtenido datos de uso de refugios mediante radioseguimiento. Dos refugios utilizados fuera de la época de cría fueron árboles de escaso porte, con huecos generados por la cicatrización de heridas longitudinales en el tronco. Localizamos también un refugio de cría, el primero en el extremo noroccidental de la Península Ibérica, situado en una grieta del tronco de un Quercus robur prácticamente muerto y de gran porte.

Palabras clave: Myotis alcathoe, Castilla y León, refugio, Ancares, Galicia.

\section{INTRODUCCIÓN}

Myotis alcathoe es una especie de reciente descripción (von Helversen y Heller 2001). Está extendida por buena parte de Europa, incluidas las Islas Británicas, ligada a bosques caducifolios maduros de valles fluviales y llanuras aluviales (Niermann et al. 2007, Lučan et al. 2009, Jan et al 2010).

Las primeras citas en el extremo noroccidental de la Península Ibérica corresponden al valle del río Eume y las sierras orientales de Galicia (Hermida y Seage, 2010; Hermida et al. 2012) y ha sido citada también en otros puntos de la mitad norte peninsular (Aguirre-Mendi et al. 2004, Alcalde, 2009; Flaquer et al. 2010).

El trabajo que se expone a continuación se enmarca dentro del proyecto "Murciélagos y soutos: un ejemplo de biodiversidad asociada a bosques culturales", con el que se dieron los primeros pasos para caracterizar el uso de hábitats y refugios de las distintas especies de murciélagos forestales que habitan las sierras de Ancares y $\mathrm{O}$ Courel. 


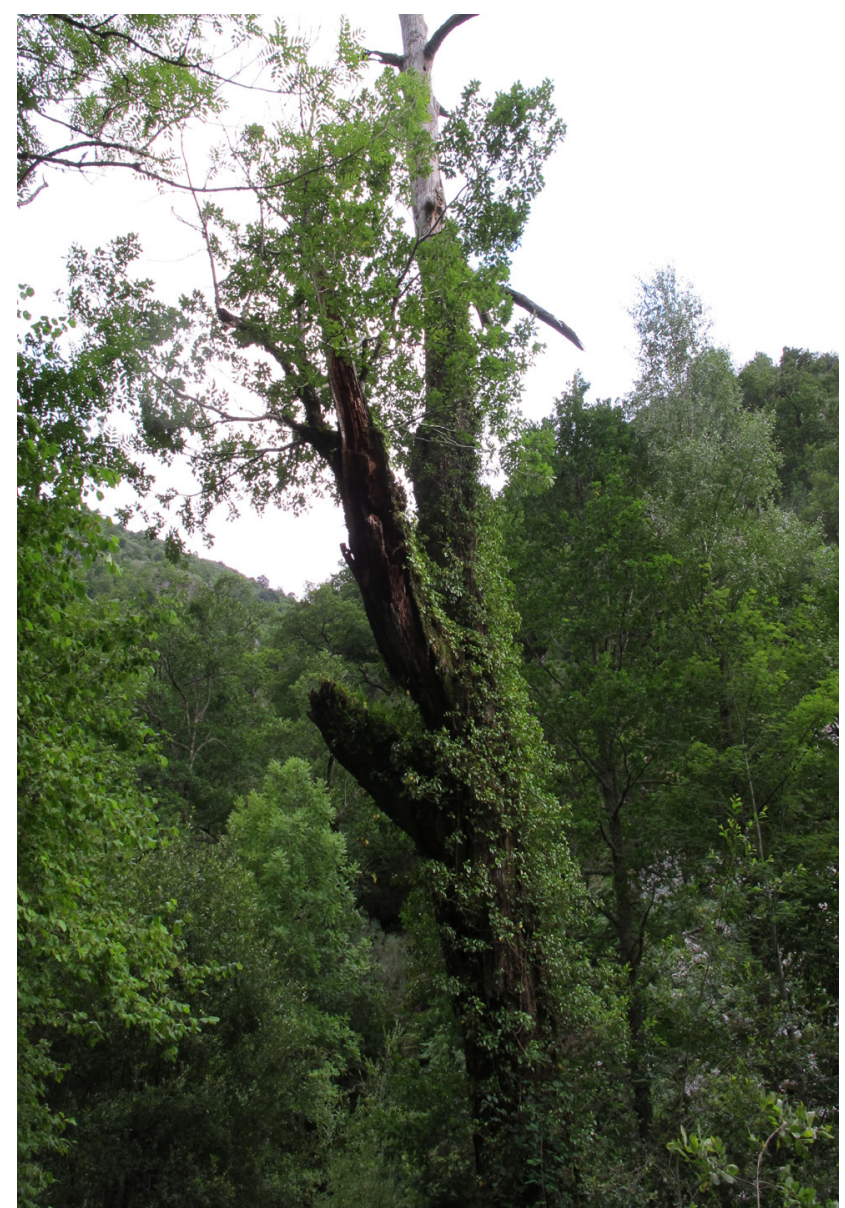

Fig 1. Vista del árbol en el que se localizó la colonia de cría.

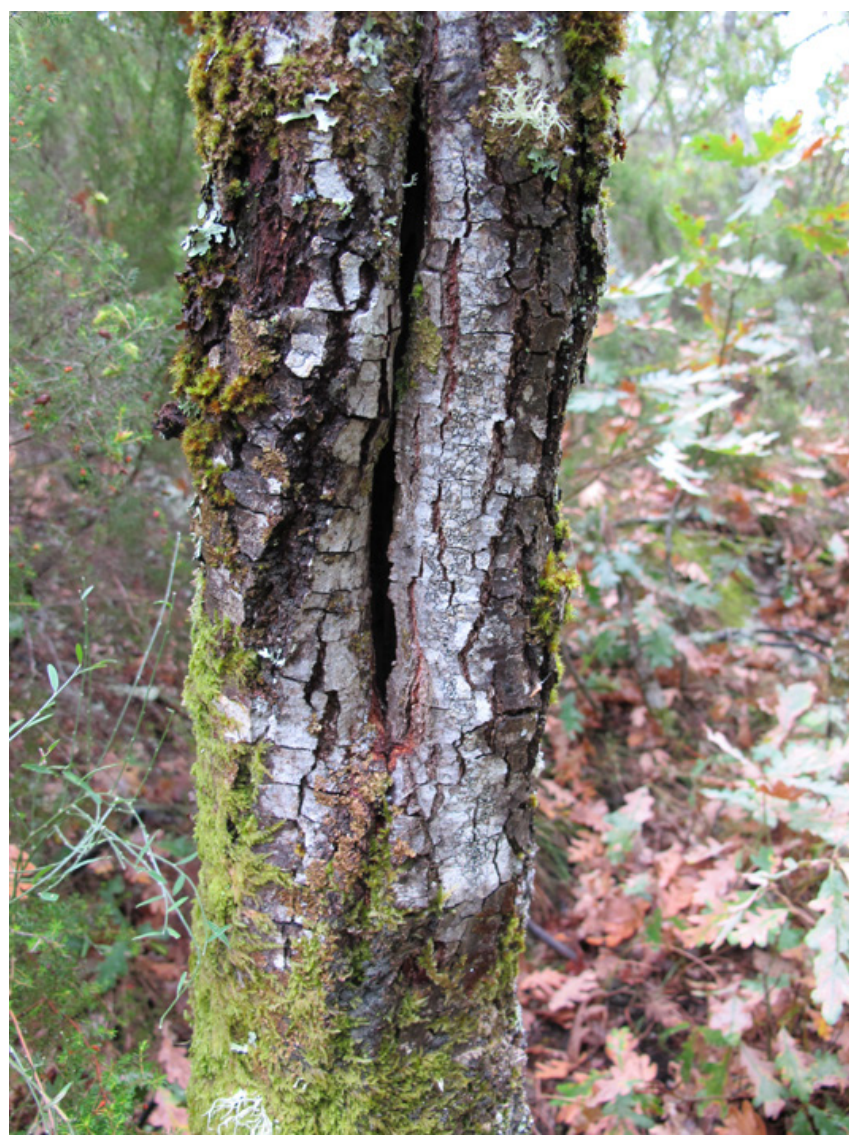

Fig 2. Árbol utilizado por el ejemplar Mal_2 en el que se aprecia la abertura del refugio.

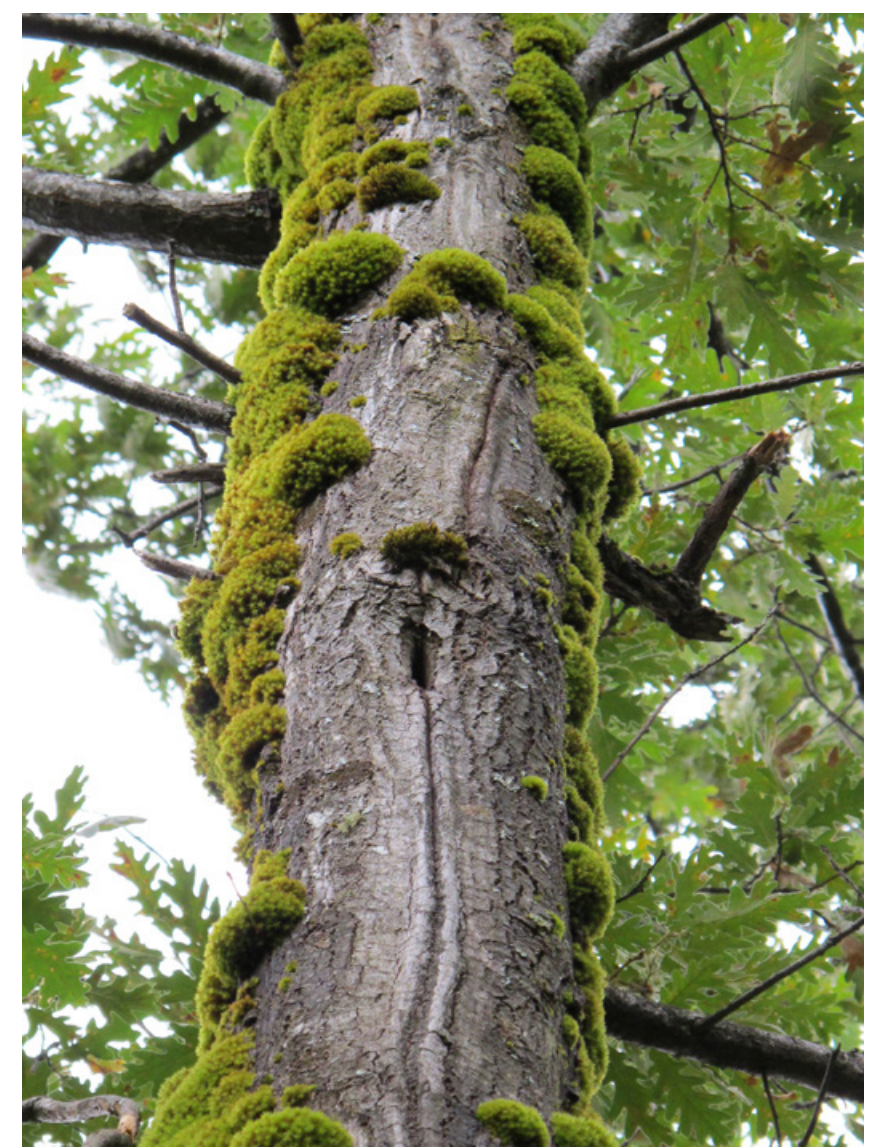

Fig 3. Detalle del refugio utilizado por el ejemplar Mal_3.

\section{Material Y MÉTODOS}

Utilizamos redes de niebla de entre 3 y 12 metros de longitud, situadas sobre cursos fluviales, para capturar todos los ejemplares. Aunque se estuvo muestreando todo el verano en tres valles distintas, solo en algunas ocasiones se colocaron redes específicamente para $M$. alcathoe. En uno de los valles solo se hicieron dos intentos, pues ya era conocida la presencia de la colonia de cría en años anteriores y no hizo falta más. En otro valle solo en una ocasión se intentó capturar la especie, con éxito. En el tercer valle, aunque se muestreó abundantemente en lugares propicios para capturar la especie ésta no cayó hasta el final del verano.

Para cada ejemplar anotamos el sexo, edad, longitud del antebrazo y peso. También se anotó cualquier signo de actividad sexual o reproductora.

La identificación específica de los individuos capturados se realizó mediante caracteres morfológicos externos (Dietz y Helversen 2004) y extrajimos muestras de tejido alar con un "biopunch" para confirmar la identificación mediante análisis de ADN mitocondrial realizados en la Estación Biológica de Doñana, (CSIC). 
Tres ejemplares fueron equipados con transmisores Pip de 0,29 g de Biotrack. Las localizaciones diurnas se realizaron por el método de homing in, utilizando dos receptores Sika de Biotrack y antenas Yagi de tres elementos. Se anotó la especie arbórea utilizada por cada ejemplar, así como otros detalles del refugio (dimensiones, naturaleza de la oquedad, altura).

En el refugio de cría se realizó una espera al anochecer en la que se contó de visu el numero de ejemplares durante la emergencia.

\section{RESULTADOS Y DISCUSIÓN}

El 22/07/2011 se capturó una hembra lactante en la localidad de Rao (Lugo), que fue equipada con un radiotransmisor. Al día siguiente se localizó la colonia de cría en una grieta situada a 14 metros de altura, en el tronco descortezado de un gran carballo (Quercus robur) prácticamente muerto, en el valle del río Balouta, en territorio leonés. Esa misma noche se realizó un conteo de ejemplares durante la emergencia crepuscular que arrojó un resultado de 22 ejemplares. Dado lo tardío de la fecha de observación, es probable que este número no se corresponda únicamente con el número de hembras adultas de la colonia de cría, sino que incluya jóvenes volantones.

Otros dos ejemplares, un macho y una hembra adultos, fueron capturados en el valle de Suertes en la vertiente leonesa de la sierra de Ancares (Candín, León) el día 31/08/2011. También fueron equipados con sendos radiotransmisores que permitieron localizarlos en refugio en los días posteriores, en las inmediaciones del punto de captura. Ambos ejemplares se refugiaron en ejemplares de Quercus pyrenaica de escaso porte, aprovechando hendiduras del tronco principal. En el caso de la hembra, la entrada del refugio estaba situada a 4,2 metros de altura, en un árbol de 72 centímetros de perímetro. Dicho refugio fue ocupado únicamente ese día. El macho ocupó, durante dos días, un hueco situado a 0,65 metros de altura, en un árbol de tan solo 30 centímetros de perímetro. Esta es la primera vez que se cita la especie en la Comunidad Autónoma de Castilla y León.

A mediados del mes de septiembre (15/09/2011) fueron capturados dos nuevos ejemplares (una hembra adulta y un macho juvenil) en un bosque limítrofe entre las provincias de Lugo y León, en la localidad de Suárbol (Candín, León).

En el mes de julio de 2009 se habían capturado en el tramo lucense del río Balouta varias hembras reproductoras de esta especie (Hermida et al. 2012) que probablemente pertenecen a la misma colonia de cría localizada en este trabajo. Según nuestra experiencia la especie se captura rápidamente cuando está presente, sobretodo en período de cría.

Tanto los puntos de captura como los datos obtenidos mediante radioseguimiento concuerdan con el uso que esta especie realiza de los paisajes que habita, moviéndose principalmente a lo largo de pequeños cursos fluviales y buscando refugio en árboles del entorno inmediato de los mismos (Lučan et al. 2009, Hermida y Seage 2010). Los refugios utilizados fuera de la época de cría también concuerdan con observaciones anteriores realizadas en Galicia en la misma época (Hermida y Seage, 2010): árboles de escaso porte con huecos generados por la cicatrización de heridas longitudinales en el tronco.

En cuanto al refugio de cría, se trata del primero localizado en el extremo noroccidental de la Península Ibérica.

Tabla 1

\begin{tabular}{llllllll}
\hline ID & Fecha captura & UTM 10x10 & Sexo & Edad & $\begin{array}{l}\text { Estado } \\
\text { reproductivo }\end{array}$ & $\begin{array}{l}\text { Antebrazo } \\
\text { (mm) }\end{array}$ & $\begin{array}{l}\text { Peso } \\
\text { (g) }\end{array}$ \\
\hline Mal_1 & $22 / 07 / 2011$ & 29 TPH75 & H & adulto & lactante & 32,1 & 5,0 \\
\hline Mal_2 & $31 / 08 / 2011$ & 29 TPH84 & M & adulto & 32,8 & 4,3 \\
\hline Mal_3 & $31 / 08 / 2011$ & 29TPH84 & H & adulto & 33,1 & 5,0 \\
\hline Mal_4 & $15 / 09 / 2011$ & 29TPH74 & M & adulto & 32,5 & 4,2 \\
\hline Mal_5 & $15 / 09 / 2011$ & 29TPH74 & M & juvenil & 31,2 & 3,7 \\
\hline
\end{tabular}




\section{Agradecimientos}

A Javier Juste, Carlos Ibáñez y Juan Luis García Mudarra, de la Estación Biológica de Doñana, por facilitarnos la identificación molecular de los ejemplares. A Francisco Conde, Sara Abalo y Zeltia López, que participaron en el trabajo de campo. Este trabajo se ha realizado con el apoyo económico de la Fundación Biodiversidad y una beca de Novacaixagalicia concedida a L.S.

Para la realización de este trabajo se ha contado con los correspondientes permisos de la Xunta de Galicia y la Junta de Castilla y León.

\section{REFERENCIAS}

Aguirre-Mendi, P.T., García-Mundarra, J.L., Juste, J. \& IBÁÑEZ, C. 2004. Presence of Myotis alcathoe Helversen and Heller, 2001 (Chiroptera: Vespertilionidae) in the Iberian Peninsula. Acta Chiropterologica 6:49-57. DOI: $\underline{\text { https://dx.doi. }}$ org/10.3161/1508110042176590

AlCALDE, J.T. 2009. Myotis alcathoe Helversen \& Heller, 2001 y Pipistrellus pygmaeus (Leach, 1825), nuevas especies de quirópteros para Navarra. Munibe, 57:225-236.

Dietz, C. \& von Helversen, O. 2004. Illustrated identification key to the bats of Europe. Electronic Publication.

Flaquer, C., Puig, X., Fàbregas, E., Guixé, D., Torre, I., RÀfols, R.G., PÁramo, F., CAmprodón, J., Cumplido, J.M., RuizJarillo, R., Baucells, A.L., Freixas, L. \& Arrizabalaga, A. 2010. Revisión y aportación de datos sobre quirópteros de Catalunya: Propuesta de lista roja. Galemys 22(1):29-61

Helversen, O. von, Heller, K.G., Mayer, F., Nemeth, A., Volleth, M. \& Gombкötä, P. 2001. Cryptic mammalian species: a new species of whiskered bat (Myotis alcathoe $\mathrm{n}$. sp.) in Europe. Naturwissenschaften 88:217223. DOI: https://dx.doi.org/10.1007/ $\underline{\mathrm{s} 001140100225}$
HeRmida, R.J. \& R. Seage (2010). Murciélagos del Parque Natural Fragas do Eume: inventario, distribución, ecología. Informe no publicado.

Hermida, R., Lamas, F., Graña, D., Rial, S., Cerqueira, F., Arzúa, M. \& Seage, R. 2012. Contribución al conocimiento de la distribución de los Murciélagos (O. Chiroptera) en Galicia. Galemys 24: 1325. DOI: https://dx.doi.org/10.7325/ Galemys.2012.A02

Jan, C.M.I., Frith, K., Glover, A.M., Butlin, R.K., Scott C.D., Greenaway, F., Ruedi, M., Frantz,A.C., Dawson, D.A. \&Altringham, J.D. 2010. Myotis alcathoe confirmed in the UK from mitochondrial and microsatellite DNA. Acta Chiropterologica 12 (2):471-483. DOI: https://dx.doi. org/10.3161/150811010X538043

Lucan R. K., Andreas M., Benda P., BartonièKa T., Bøezinová, T., Hoffmannová A., Hulová, Hulva P., Neckaøová J., Reitera., SvaÈINA T., ŠÁLEK M. \& HorÁÈEK, I. 2009. Alcathoe bat (Myotis alcathoe) in the Czech Republic: distributional status, roosting and feeding ecology . Acta Chiropterologica 11(1):61-69. DOI: https:// dx.doi.org/10.3161/150811009X465695

Niermann I., Biederman M., Bogdanowicz W., Brinkman R., Le Bris Y., Ciechanowski M., Dietz C., Dietz I., Estók P., von Helversen O., Le Houédec A., Paksuz S., Petrov B., ÖZKanB.,PiksaK., RachwaldA.,RouÉS.Y., SACHANOWICZ K., Schorcht W., TerebaA.\& MAYER F. 2007. Biogeography of the recently described Myotis alcathoe von Helversen and Heller, 2001. Acta Chiropterologica 9(2):361-378. 